\title{
Générer des impulsions laser ultra-brèves de très haute intensité : la technique du CPA
}

Chirped pulse amplification ou amplification par dérive de fréquence

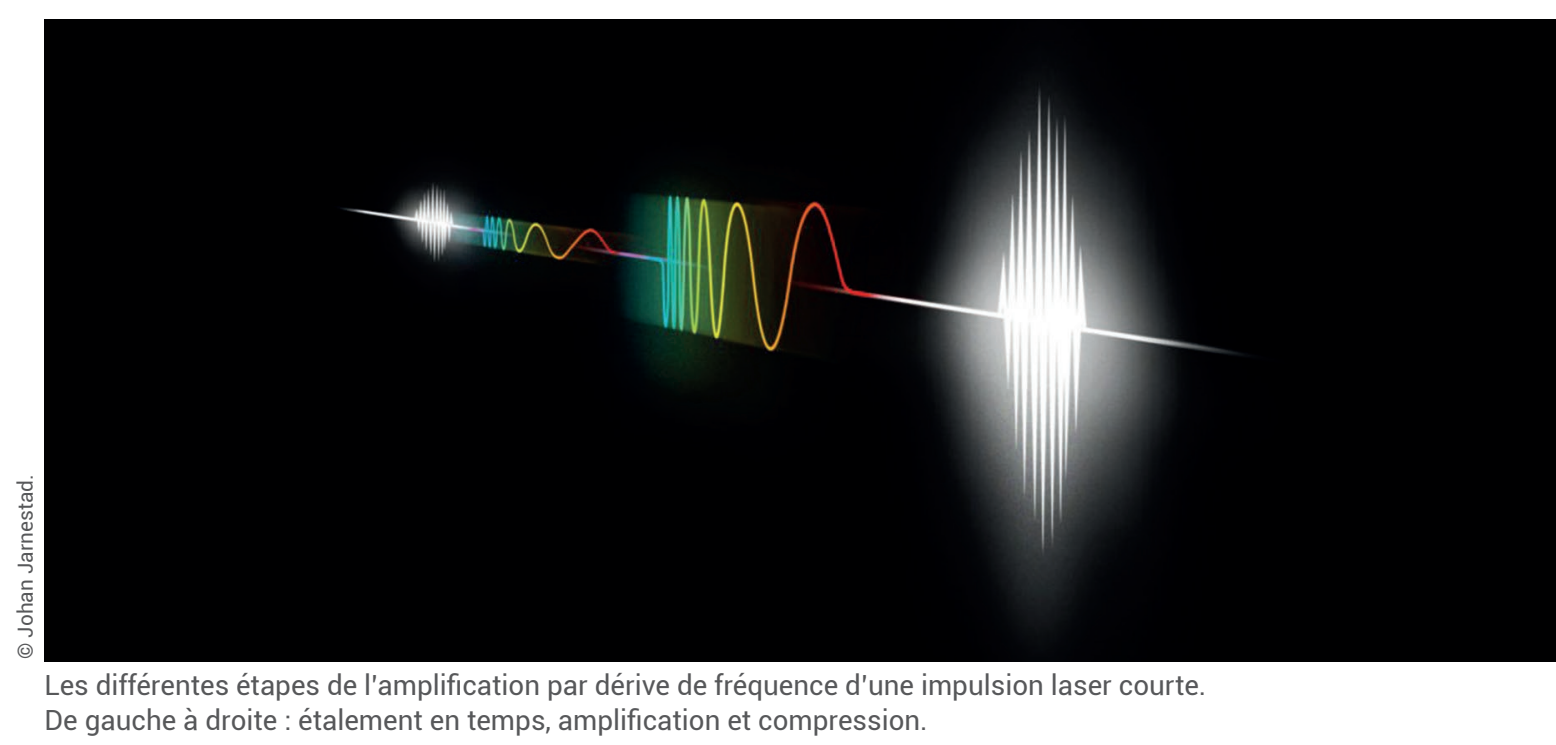

Il y a un avant et un après l'article publié en 1985 par Donna Strickland et Gérard Mourou [1] faisant la démonstration de l'amplification par dérive de fréquence (CPA pour Chirped Pulse Amplification).

Le présent dossier de Reflets de la physique a pour but de donner une vue d'ensemble de cette technologie et de ses différentes implications. Le principe de l'amplification par dérive de fréquence est d'abord présenté, avec son développement dans le monde des lasers.

Ensuite, sont décrites les applications industrielles relatives au micro-usinage et à l'ophtalmologie, qui concernent les intensités moyennes $\left(10^{13}-10^{14} \mathrm{~W} \mathrm{~cm}-2\right)$.

Les applications à la recherche couvrent les intensités plus fortes $\left(10^{14}-10^{18} \mathrm{~W} \mathrm{~cm}^{-2}\right)$, qui permettent la génération d'impulsions ultra-courtes et de sources nouvelles de photons et de particules, jusqu'à la ultra-haute intensité $\left(10^{18}-10^{22} \mathrm{~W} \mathrm{~cm}^{-2}\right.$ et au-delà...) et les lasers multipétawatts.

Dans le tableau ci-contre, nous rappelons les préfixes du système international d'unités, qui désignent des multiples ou des fractions de 10. Ces préfixes simplifient la manipulation des valeurs numériques des grandeurs physiques qui sont beaucoup plus petites ou plus grandes que l'unité officielle.

\begin{tabular}{c|c|c|c}
$\begin{array}{c}\text { nano }(\mathrm{n}) \\
10^{-9}\end{array}$ & $\begin{array}{c}\text { pico }(\mathrm{p}) \\
10^{-12}\end{array}$ & $\begin{array}{c}\text { femto }(\mathrm{f}) \\
10^{-15}\end{array}$ & $\begin{array}{c}\text { atto }(\mathrm{a}) \\
10^{-18}\end{array}$ \\
\hline giga $(\mathrm{G})$ & téra $(\mathrm{T})$ & péta $(\mathrm{P})$ & exa $(\mathrm{E})$ \\
$10^{9}$ & $10^{12}$ & $10^{15}$ & $10^{18}$
\end{tabular}




\title{
Principe et développements
} de la technique du CPA

\author{
Frédéric Druon (frederic.druon@institutoptique.fr) \\ Laboratoire Charles Fabry (CNRS/IOGS), 2 avenue Augustin Fresnel, 91127 Palaiseau Cedex
}

Un des grands enjeux récurrents dans le développement des lasers depuis leur invention est, sans nul doute, l'augmentation de l'intensité lumineuse, avec pour objectif d'accéder à des régimes d'interaction lumière-matière de plus en plus extrêmes. Les lasers à impulsions ultrabrèves (picosecondes et femtosecondes) représentent un excellent moyen pour $y$ parvenir, car ils permettent d'obtenir de très fortes puissances crêtes en concentrant dans un temps très court $(\Delta \tau)$ une énergie lumineuse (E) dans la gamme typiquement $\mathrm{du}$ millijoule à quelques centaines de joules. En focalisant le faisceau laser sur une petite surface (S), il est alors possible d'obtenir de très hautes intensités - ou éclairements du point de vue photométrique (voir la partie sur les intensités fortes, pp. 20-24) : I = E / (S $\Delta \tau)$.

Dans les années 1970, la montée en puissance des lasers a subi un fort ralentissement. En effet, la réduction de la durée des impulsions - qui était intrinsèquement limitée par les milieux laser dopés au néodyme $(\mathrm{Nd})$ utilisés à l'époque ${ }^{(\mathrm{a})}$ - avait atteint une limite, et le seul moyen d'augmenter la puissance crête consistait à accroitre l'énergie. La montée en énergie passe par une étape d'amplification laser qui consiste à faire traverser au faisceau une séquence de milieux à gain laser. Or, la tenue au flux lumineux des matériaux étant limitée (typiquement à quelques $\mathrm{GW} \mathrm{cm}^{-2}$ ), l'endommagement des optiques, notamment des milieux amplificateurs, a rapidement été un verrou technologique bloquant. Ainsi, avant l'invention de la technique du CPA, pour pallier cette limitation, la seule solution était d'agrandir suffisamment les faisceaux dans les milieux amplificateurs afin d'éviter tout risque d'endommagement. Pour atteindre des puissances crêtes de plusieurs TW, des lasers gigantesques ont été alors développés. Ces grands instruments avaient une exploitation difficile et étaient réservés à quelques expériences scientifiques. Dans ce cadre, l'amplification par dérive de fréquence (ou CPA pour Chirped Pulse Amplification) a été un changement de paradigme radical, et les travaux fondateurs de Donna Strickland et de Gérard Mourou [1] ont, comme nous allons le voir, ouvert une nouvelle ère dans le développement des lasers intenses.

\section{Principe de l'amplification par dérive de fréquence}

Le principe de base de la technique du CPA consiste à transposer dans le domaine temporel ce qui est fait dans le domaine spatial pour gérer la dimension des faisceaux afin d'éviter les dommages dans les amplificateurs. Cette idée, déjà envisagée par les spécialistes des radars, commence à apparaitre dans les années 1960 - notamment dans les travaux de Pierre Tournois [2]. Elle consiste à étaler dans le temps les différentes longueurs d'onde d'une impulsion et à créer ainsi une dérive de fréquence en fonction du temps, tel le gazouillis des oiseaux (chirp en anglais) qui glisse des sons graves aux aigus. Pour cela, il suffit de faire parcourir à chaque composante spectrale de l'impulsion une longueur optique différente. Cela allonge de facto la durée de l'impulsion et réduit son intensité (fig. I.1). La technique du CPA intègre donc des éléments spectralement dispersifs afin d'imposer un délai différent - et contrôlé - pour chacune des longueurs d'onde ${ }^{(b)}$. Elle va comporter trois étapes, consistant d'abord à étirer temporellement les impulsions, puis à les amplifier dans des matériaux laser sans risquer l'endommagement et, enfin, à les comprimer pour retrouver la durée initiale (idéalement) et une forte intensité (fig. I.1). Pour y parvenir, la technique du CPA utilise astucieusement une propriété particulière des impulsions courtes : leur spectre large. En effet, la forme temporelle et le spectre des impulsions étant liés par transformée de Fourier, il existe une relation entre la durée de l'impulsion et la largeur spectrale. Plus une impulsion est brève, plus son spectre est large et, par conséquent, plus il est possible d'étaler ses différentes composantes spectrales. Pour un spectre donné, si toutes les longueurs d'onde se propagent sans délai les unes par rapport aux autres (pas de dérive de fréquence), la durée de l'impulsion est la plus courte envisageable. Dans le cas contraire, si les temps de propagation varient d'une longueur d'onde à l'autre, l'impulsion aura une dérive de fréquence et sera de durée plus longue.

\section{Étirer et comprimer}

La manipulation de la dérive de fréquence des impulsions courtes est donc au cœur de la technique du CPA. L'idée forte est d'avoir trouvé un moyen réversible pour étirer puis comprimer temporellement et de manière substantielle les impulsions. En effet, autant l'étirement des impulsions à large spectre est observé couramment car tout matériau traversé présente un indice optique dépendant de la longueur d'onde, ce qui crée naturellement une variation des vitesses de propagation avec la longueur d'onde et donc une dérive de fréquence, autant la recompression est plus délicate. Ainsi, dans la première expérience de Strickland et Mourou, une fibre optique de 1,4 km a été utilisée pour permettre une forte dispersion chromatique dite normale : les basses fréquences de l'impulsion (dites longueurs d'onde " rouges ") ont un trajet optique plus court et sont, à la sortie de la 


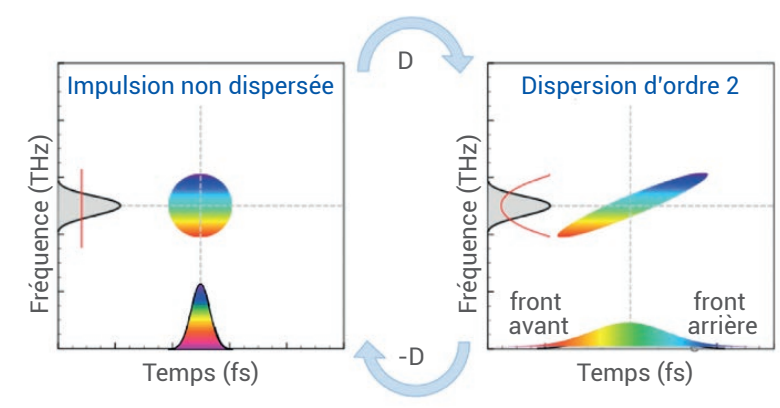

Opération réversible

fibre, en avance par rapport aux hautes fréquences (dites longueurs d'onde «bleues»). Cependant, aucun matériau avec une dispersion chromatique inverse (c'est-à-dire anormale) suffisante n'était disponible. Pour pallier cette difficulté, un système à réseaux (proposé par Treacy en 1969 [3]), permettant de faire parcourir des distances différentes selon les longueurs d'onde et d'imprimer une forte dérive de fréquence de type anormale, a été mis en place (fig. I.2). La première expérience d'amplification à dérive de fréquence démontrée en 1985 [1] intégrait donc un générateur d'impulsions picoseconde (appelé oscillateur), une fibre optique permettant un facteur d'étirement de deux ordres de grandeur, un amplificateur laser ${ }^{(c)}$ et un compresseur à réseaux.

\section{Vers les hautes intensités}

La technique du CPA a offert une réelle rupture technologique avec des conséquences importantes sur le développement des lasers impulsionnels. Tout d'abord, des lasers de très forte puissance crête de type térawatt (TW) ont pu être développés sur des espaces réduits. On parle alors de Table-Top TW $\left(\mathrm{T}^{3}\right)$ lasers. Ceci a permis une dissémination des lasers de très forte intensité dans de nombreux laboratoires et a ouvert la voie à de nombreuses expériences de physique fondamentale grâce à ces nouveaux lasers multi-TW [4] puis pétawatts (PW) [5]. En effet, les lasers ultra-intenses ont, de surcroît, bénéficié de deux améliorations notables dans les années 1980 : d'une part, une amélioration des systèmes étireurs en remplaçant les fibres par des étireurs à réseaux de type Martinez [6], ce qui a permis une compression temporelle de meilleure qualité (fig. I.3) et, d'autre part, l'apparition du cristal saphir dopé au titane $\left(\mathrm{Ti}^{3+}: \mathrm{Al}_{2} \mathrm{O}_{3}\right.$ ou $\left.\mathrm{Ti}: \mathrm{Sa}\right)$ [7]

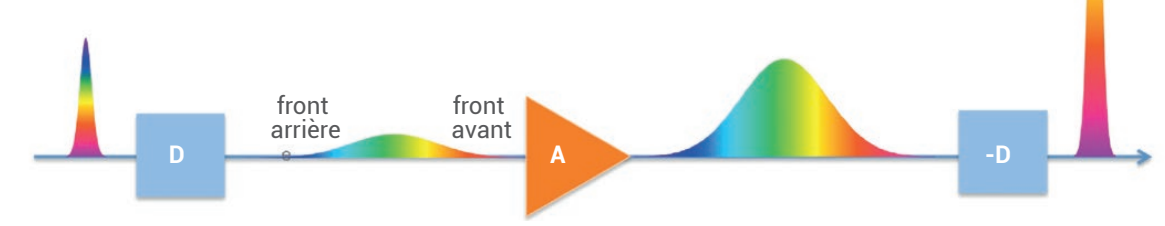

I.1. Schéma de l'amplification par dérive de fréquence. La technique du CPA comporte trois étapes : étirement, amplification et compression des impulsions. Le spectre d'une impulsion courte peut être étalé temporellement en lui imposant une dérive de fréquence en fonction du temps. Les deux spectrogrammes du haut montrent l'évolution du spectre en fonction du temps pour les cas sans (à gauche) et avec (à droite) dérive linéaire de fréquence. Sur l'axe des ordonnées sont représentés, en noir, le spectre (qui est le même dans les deux cas) et, en rouge, la phase spectrale associée. Sur l'axe des abscisses est représentée la forme temporelle de l'impulsion. Quand l'impulsion n'est pas dispersée, toutes les composantes spectrales sont synchrones et l'impulsion est la plus courte et intense possible. A contrario, les différentes longueurs d'onde peuvent être dispersées temporellement avec une dérive de fréquence typiquement linéaire (ici les longueurs d'onde rouges arrivent avant les bleues) et, pour le même spectre, la durée est plus longue et l'intensité plus basse. Cet effet permet de réduire la puissance crête d'un facteur égal à celui d'étirement. Cette dispersion chromatique est réversible en fonction du signe de la dispersion. Le CPA est donc composé de trois opérateurs (image du bas) : un opérateur dispersion ( $D$, avec typiquement $D<0$ pour avoir les longueurs d'onde rouges en avance par rapport aux bleues) nommé " étireur ", un opérateur amplificateur optique typiquement laser (A) et un opérateur dispersion opposé (-D) nommé « compresseur ». Les facteurs d'étirement (c'est-à-dire de réduction de puissance crête) sont dans les lasers PW actuels de l'ordre de 100000.

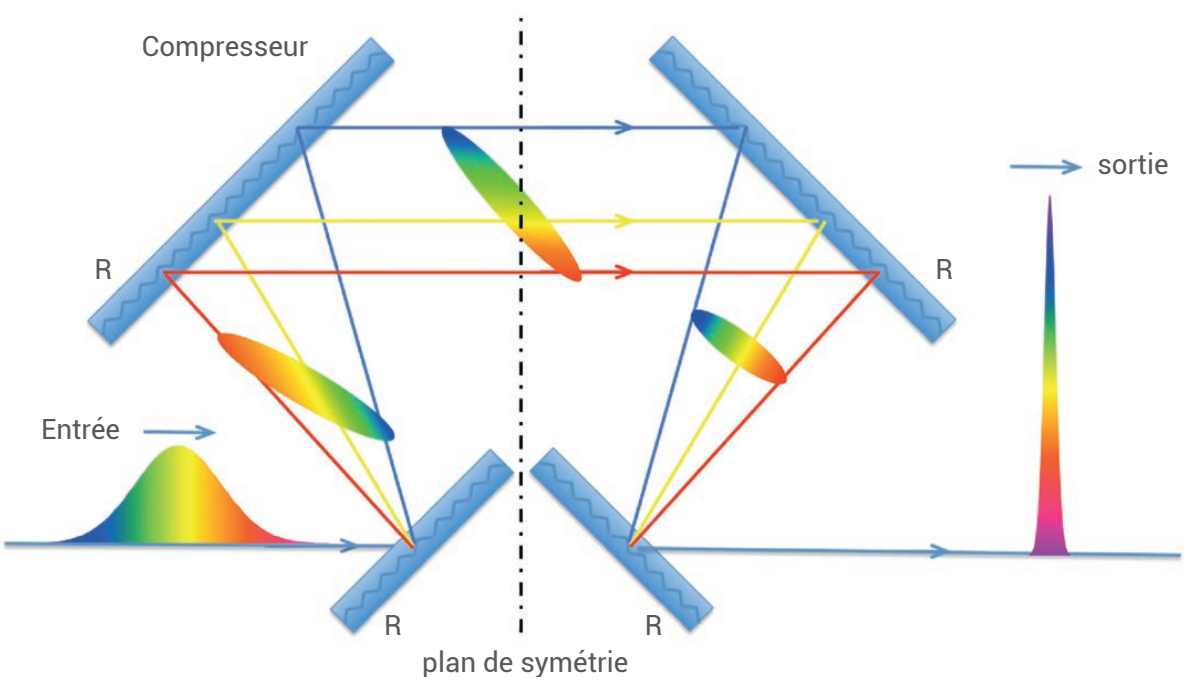

I.2. Schéma du compresseur à réseaux (opérateur -D sur la figure I.1). Le premier réseau (en bas, à gauche) permet une transformation spectre-angle. Le second réseau (en haut, à gauche), parallèle au premier et aux mêmes caractéristiques, permet une transformation spectre-position. Le système est ensuite symétrisé pour recombiner spatialement les différentes composantes spectrales. (N.B. : le compresseur à réseaux peut être réduit à deux réseaux par utilisation d'un miroir dans le plan de symétrie, qui permet de renvoyer le faisceau dans le sens inverse.) En sortie du compresseur, les différentes longueurs d'onde sont à nouveau spatialement superposées, mais après avoir effectué des trajets différents : plus long pour le rouge que pour le bleu. 
qui, grâce à une bande d'amplification extrêmement large (permettant l'amplification d'impulsions de quelques dizaines de femtosecondes), a commencé à supplanter les lasers à base de verres dopés au néodyme. Les CPA ont ainsi vu leur facteur d'étirement notablement amélioré jusqu'à atteindre actuellement des valeurs typiquement de $10^{5}$, démontrant alors l'intérêt indéniable de cette technique pour les lasers ultra-intenses.

\section{Les développements industriels}

Une autre conséquence importante du CPA concerne le développement de lasers CPA moins énergétiques mais ultracompacts, avec des puissances crête de l'ordre du GW [8]. Ce nouveau type de lasers a rapidement prouvé un très fort potentiel d'industrialisation. Ils sont, en effet, suffisamment intenses $\left(10^{13}-10^{14} \mathrm{~W} \mathrm{~cm}^{-2}\right)$ pour permettre une découpe athermique de très grande qualité (voir l'article de M. Sentis, p.17). Ces lasers intenses ont alors été intégrés dans des outils ophtalmologiques parmi lesquels on peut citer, comme application phare, la découpe de la cornée pour la chirurgie réfractive [9] dont la société IntraLase a été pionnière dès le début des années 1990 (voir l'article de F. Salin, p.18).

L'amplification par dérive de fréquence est une technologie vivante. Elle a été intégrée dans de multiples systèmes amplificateurs comme les lasers à base de matériaux dopés à l'ytterbium $(\mathrm{Yb})$ pompés par diode et, en particulier, les fibres amplificatrices dopées $\mathrm{Yb}$ (YDFA). Ces systèmes laser permettent d'obtenir des hauts taux de répétition (de $100 \mathrm{kHz}$ à quelques $\mathrm{MHz}$ typiquement) et des puissances moyennes importantes (pouvant aller jusqu'au $\mathrm{kW}$ ). Ils sont donc extrêmement utilisés dans l'industrie. On peut aussi noter les nombreuses avancées sur les étireurs et compresseurs, avec des systèmes pour manipuler la dérive de fréquence de plus en plus précis et compacts (fibres de Bragg, miroirs diélectriques à dispersion contrôlée, réseaux de Bragg très efficaces, modulateurs acousto-optiques programmables, etc.). La technique du CPA a donc gagné en maturité pour devenir primordiale et omniprésente sur le marché des lasers industriels intenses.

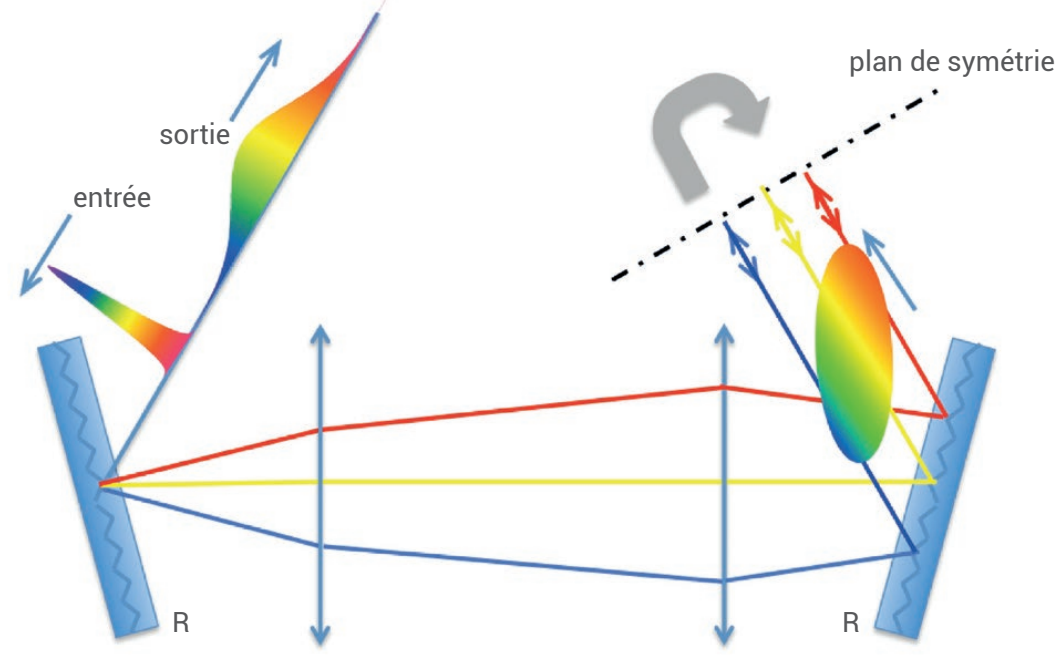

I.3. Schéma d'un étireur à réseaux (opérateur D sur la figure I.1). L'étireur à réseaux utilise un système d'imagerie constitué de lentilles (ou de miroirs) afin de réaliser un délai entre les différentes longueurs d'onde, le plus exactement possible inverse à celui du compresseur. Tout comme pour le compresseur, le faisceau passe quatre fois dans les réseaux, étant renvoyé dans le sens inverse au niveau du plan de symétrie. Après un aller-retour, les différentes longueurs d'onde sont spatialement superposées, mais avec des trajets différents : plus court pour le rouge que pour le bleu. Du point de vue de l'optique géométrique, l'étireur à réseaux peut être considéré comme rigoureusement le système inverse du compresseur à réseaux. La compression de la dérive de fréquence est ensuite de bien meilleure qualité comparée aux premiers étireurs à fibres utilisés qui ne permettent pas cette compensation parfaite de la dérive, notamment à cause des termes d'ordre supérieurs.

\section{L'amplification paramétrique optique}

La technique du CPA a aussi été étendue à d'autres méthodes d'amplification nonlaser comme l'amplification paramétrique optique (OPA), avec les systèmes dits OPCPA (association des sigles OPA et CPA). L'OPA est un effet non linéaire qui consiste à amplifier, à l'aide d'un laser de pompe, deux ondes - respectivement " signal " et " complémentaire " - dont la somme des fréquences est égale à la fréquence du laser de pompe. L'OPA permet ainsi d'amplifier des longueurs d'onde où les transitions laser font défaut. À l'inverse du laser, l'OPA est un phénomène instantané et il permet une amplification temporellement plus ciblée et un meilleur contraste temporel. Ce dernier définit la pureté de l'impulsion : il est en effet important qu'il n'y ait pas de piédestal avant l'impulsion, car l'énergie qu'il contient peut donner lieu à une interaction lumière-matière avant même l'arrivée de l'impulsion principale et donc modifier radicalement les interactions visées. Ces systèmes OPCPA sont donc particulièrement utilisés, soit pour accéder à de nouvelles longueurs d'onde, soit pour assurer le très bon contraste temporel que demandent les systèmes de classe pétawatts [10].

En conclusion, l'amplification par dérive de fréquence a intégré de nombreuses améliorations et extensions technologiques qui ont permis une dissémination encore plus large des lasers ultra-intenses avec, comme on va le voir dans la suite, de multiples applications industrielles et scientifiques.

(a) La limitation en durée est de quelques centaines de femtosecondes pour les verres dopés au $\mathrm{Nd}$ et de quelques picosecondes pour les cristaux dopés au $\mathrm{Nd}$.

(b) La dérive de fréquence en fonction du temps est typiquement linéaire. Elle correspond par intégration à une phase spectrale parabolique.

(c) L'amplificateur utilisé dans cette expérience était de type régénératif : il consistait à piéger, par des jeux de polarisation, une impulsion dans une cavité laser pour la faire passer un grand nombre de fois dans le milieu laser et ainsi obtenir un fort gain. 


\title{
Intensités moyennes: \\ applications industrielles
}

\section{Les applications au micro-usinage}

\author{
Marc Sentis (marc.sentis@univ-amu.fr) \\ Laboratoire Lasers, plasmas et procédés photoniques (CNRS/Aix-Marseille Université), \\ 163 Avenue de Luminy, 13009 Marseille
}

À l'origine de la technique d'usinage par laser, il y a la focalisation d'un faisceau laser infrarouge (laser à $\mathrm{CO}_{2}$ ou verres dopés au $\mathrm{Nd}$ par exemple) sur un matériau pour permettre la sublimation ou l'évaporation de matière par effet thermique. Dès la fin des années 1960, le terme "microusinage laser " apparait dans des publications et brevets. Plus largement, le terme générique micro-usinage est utilisé pour désigner un processus permettant de créer des éléments de taille micrométrique. En utilisant un laser impulsionnel, il devient possible de déposer de petites quantités d'énergie en un temps court, ce qui permet de réduire la longueur de diffusion thermique (plus la durée d'impulsion est courte, plus la longueur de diffusion thermique est petite) et d'obtenir un enlèvement de matière précis et reproductible. Ce procédé laser permet donc de découper, percer, graver et de manière générale d'usiner un matériau à de très faibles échelles spatiales, inférieures au micromètre. De plus, par rapport aux techniques traditionnelles de découpe, c'est un procédé sans contact. Nous verrons comment les impulsions ultra-courtes femtoseconde ont permis de passer du micro-usinage au nano-usinage.

Les paramètres laser importants pour optimiser le procédé de micro-usinage laser sont la longueur d'onde, la durée d'impulsion, l'énergie délivrée par impulsion, et la puissance moyenne qui est reliée au taux de répétition du laser et à l'énergie par impulsion.

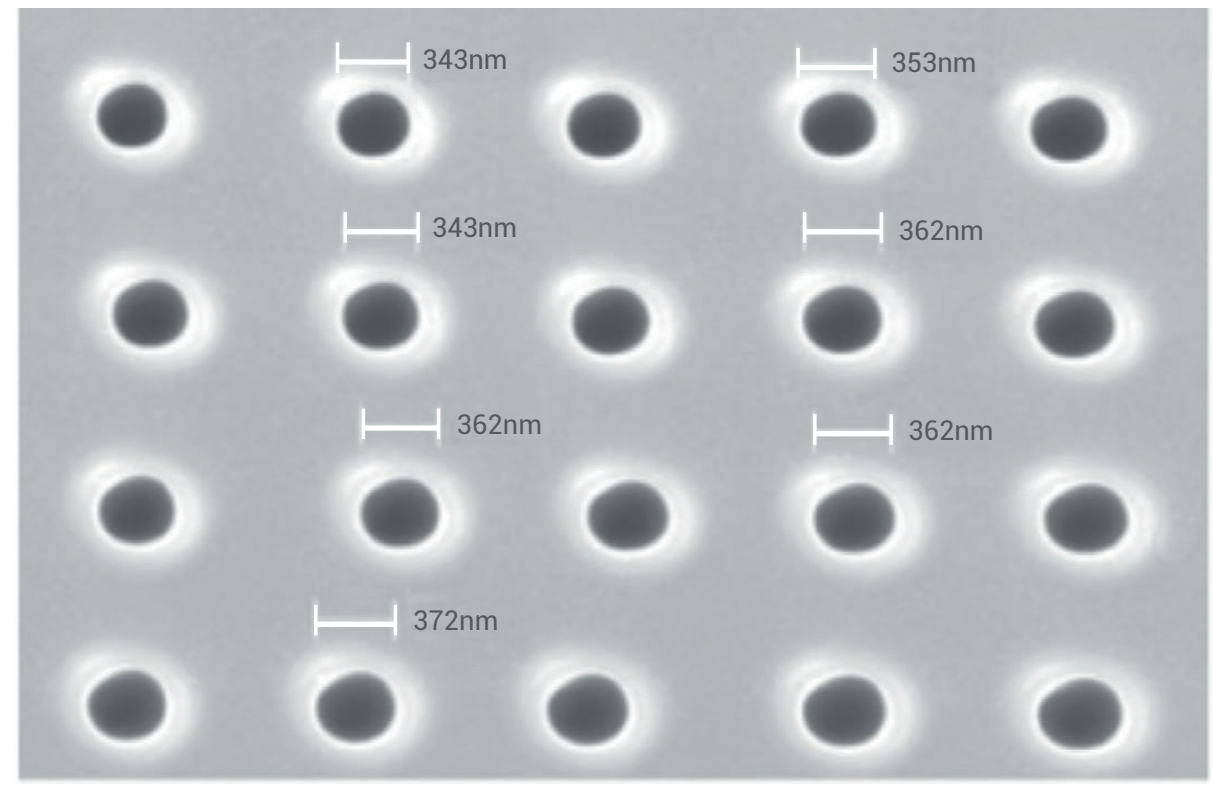

II. 1. Matrice de nanotrous dans du verre, réalisée par ablation laser $(\lambda=1053 \mathrm{~nm}, \tau=600 \mathrm{fs})$ [12]. Les trous ont une dimension de l'ordre de $350 \mathrm{~nm}$ (mesures entre 343 et $372 \mathrm{~nm}$ ). De telles dimensions, nettement inférieures à la limite de diffraction, sont obtenues grâce aux impulsions femtoseconde intenses pour lesquelles l'ablation est contrôlée par des effets non linéaires.

La longueur d'onde est importante, car elle intervient à la fois sur la précision de l'usinage et sur son rendement. Plus la longueur d'onde sera courte, plus il sera facile de focaliser le laser sur une surface de très petite taille en s'approchant de la limite de diffraction. Par ailleurs, le coefficient d'absorption du matériau dépend de la longueur d'onde, déterminant ainsi sur quelle profondeur le rayonnement laser va pénétrer. À titre d'exemple, les lasers à excimère $^{(a)}$, qui émettent dans le domaine de l'ultraviolet (UV), ont été et sont utilisés pour micro-usiner avec une grande précision de nombreux matériaux.
Comparés aux photons infrarouges, les photons UV ont une énergie plus élevée qui permet, comme dans le cas des polymères, la photo-ablation, c'est-à-dire la rupture des liaisons sans échauffement du matériau.

La durée de l'impulsion ( $\boldsymbol{\tau}$ ) est également un paramètre crucial : durée et énergie par impulsion sont étroitement liées pour permettre d'atteindre le seuil d'ablation d'un matériau (exprimé en $\mathrm{J} \mathrm{cm}^{-2}$ ). Dans le cas des métaux par exemple, ce seuil est de l'ordre de $0,1 \mathrm{~J} \mathrm{~cm}^{-2}$ pour des impulsions femtoseconde, soit environ dix fois moindre que pour des impulsions nanoseconde. 
Pour un matériau diélectrique tel que le verre, transparent dans un large domaine spectral, l'absorption linéaire est faible et son micro-usinage sans effets secondaires devient difficile avec des impulsions laser de durée nanoseconde ou plus longue. L'ablation du matériau est alors fortement dépendante des électrons libres piégés dans les défauts et les impuretés. Mais, dès que l'intensité de l'impulsion devient supérieure à $\sim 10^{13} \mathrm{~W} \mathrm{~cm}^{-2}$, le processus d'ablation entre dans un régime d'interaction très différent : des processus non linéaires tels que l'ionisation multiphotonique et/ou par effet tunnel, ainsi que le mécanisme d'avalanche, permettent une ionisation locale efficace du matériau. Ces régimes d'intensité sont devenus très facilement accessibles avec l'introduction du CPA dans les lasers femtoseconde. Le processus d'ablation devient alors fortement déterministe [11], ce qui veut dire que les seuils d'ablation sont définis très précisément et qu'ainsi l'ablation devient hautement contrôlable. De plus, la zone affectée thermiquement devient extrêmement réduite, d'où l'introduction dans les années 1990 du terme "ablation froide ". Travailler à ce niveau d'intensité avec des impulsions laser ultracourtes rend possible de micro-usiner pratiquement tous les matériaux, y compris le diamant, avec une qualité de précision dimensionnelle, de reproductibilité, de limitation de bavures et d'effets thermiques difficilement atteignable par d'autres techniques. La figure II.1 est une illustration de la précision et de la reproductibilité d'un tel procédé jusqu'à des échelles submicroniques [12].

Enfin, à l'échelle industrielle, la puissance moyenne du laser est importante pour réaliser des micro-usinages dans un temps économiquement viable. Très peu de temps après l'invention du CPA, les fabricants de lasers se sont attachés à utiliser cette technique pour augmenter la puissance moyenne, tout en rendant les sources plus efficaces et plus compactes. Cette course à la compacité et à la puissance moyenne est toujours d'actualité, et presque toutes les approches utilisées sont basées sur le CPA dès que l'on dépasse quelques watts.

(a) Les lasers à excimère (contraction des mots anglais "excited" et "dimer") sont des lasers dont le milieu actif gazeux est composé d'un gaz rare (Xe, Kr, Ar) et d'un halogène $(\mathrm{Cl}, \mathrm{F})$. Ils émettent dans le domaine de l'UV (193 nm pour ArF, $308 \mathrm{~nm}$ pour XeCl...). Leur nom vient du fait que ces molécules ne sont stables qu'à l'état excité, l'état fondamental étant dissociatif.

\section{Les applications à l'ophtalmologie}

François Salin (francois.salin@ilasis.com)

Ilasis Laser, 2 allée du Doyen Georges Brus, 33600 Pessac

L'application des lasers femtoseconde à l'ophtalmologie a commencé par un accident qui, heureusement, s'est bien fini et a ouvert un champ très important de la chirurgie oculaire. En 1992, un chercheur du Center for Ultrafast Optical Science (CUOS) que dirigeait Gérard Mourou à l'Université du Michigan, a reçu accidentellement un tir de laser femtoseconde sur la rétine. Se rendant au Kellogs Eye Center d'Ann Arbor pour un diagnostic, il excite la curiosité du médecin qui l'examine par la propreté de l'impact laser dans l'œil et l'absence de dégâts autour du point focal. La spécificité ainsi révélée est que l'interaction d'une impulsion femtoseconde dans un milieu transparent est extrêmement localisée. En effet, il est possible de créer un défaut - une microbulle - en focalisant fortement un faisceau femtose- conde à l'intérieur du milieu. Au foyer, l'intensité atteint des valeurs supérieures à $10^{13} \mathrm{~W} \mathrm{~cm}^{-2}$, ce qui provoque une ionisation multiphotonique de l'eau présente au sein des tissus : on voit alors apparaitre une microbulle de quelques microns de diamètre. Comme l'ouverture numérique du faisceau dépasse généralement 0,2 (soit environ 20 degrés), la bulle est localisée non seulement latéralement, mais aussi en profondeur. En déplaçant le faisceau sur l'ensemble de la surface d'un disque de 8 mm de diamètre, on crée ainsi un plan de bulles microscopiques (fig. II.2) qui ont le même effet qu'un scalpel, mais à l'intérieur de la matière et sans endommager les surfaces autour du point d'impact. Ce principe de découpe très localisée de tissus transparents est spécifique aux lasers femtoseconde.

\section{Correction de la myopie par laser}

La myopie correspond à un défaut de sphéricité de l'œil. Ce défaut se corrige en ouvrant un " capot " très fin (de l'ordre de $100 \mu \mathrm{m}$ d'épaisseur) à la surface de la cornée, puis en sculptant la cornée (de l'ordre de $600 \mu \mathrm{m}$ au point le plus épais), c'est-à-dire en retirant l'épaisseur de matière nécessaire pour corriger le défaut à l'aide d'un laser excimère, avant de repositionner le capot. Cette correction a typiquement la forme d'une petite lentille d'environ $8 \mathrm{~mm}$ de diamètre et de $100 \mu \mathrm{m}$ d'épaisseur au centre et $0 \mu \mathrm{m}$ sur les bords.

La société IntraLase fut créée en 1995 à Ann Arbor pour appliquer les lasers femtoseconde pompés par diodes, qui commen- 

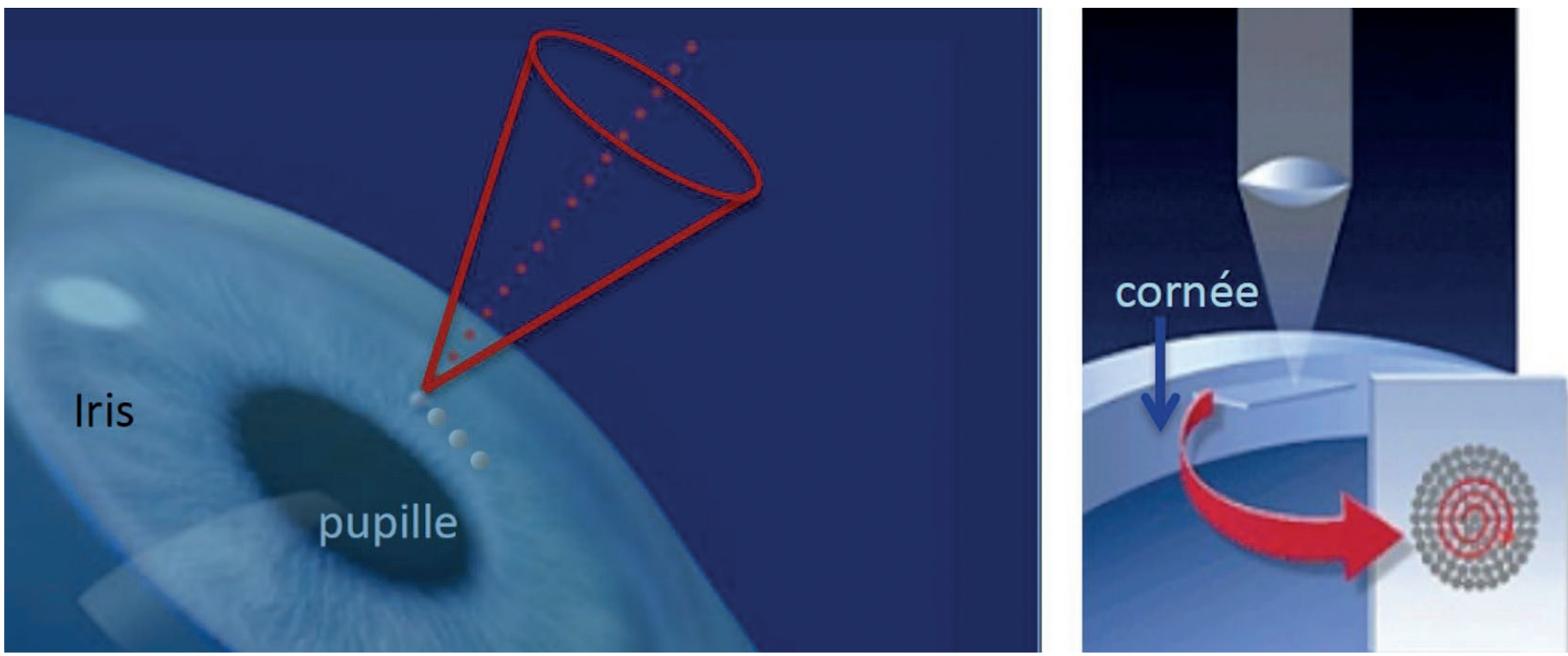

II.2. Création par laser femtoseconde d'un plan de bulles localisé dans la cornée. La figure de gauche montre de façon schématique l'ouverture du faisceau laser (en rouge) et la formation de quatre bulles dans les $600 \mu \mathrm{m}$ de l'épaisseur de la cornée (la cornée est située environ $3 \mathrm{~mm}$ au-dessus de l'iris et de la pupille). À droite, on voit le disque de bulles d'une épaisseur de quelques microns formé par balayage du faisceau laser, et se situant à environ $100 \mu \mathrm{m}$ de profondeur dans la cornée (représentée par la lame claire courbée). À la fin du balayage, ce disque aura un diamètre de $8 \mathrm{~mm}$, légèrement plus grand que celui de la pupille quand elle est très dilatée, pour que les bords du cercle découpé ne gênent pas la vision.

çaient à voir le jour au CUOS, à la découpe intracornéenne avec la technique Lasik (Laser-Assisted In-Situ Keratomileusis) [9, 13]. Les premiers lasers utilisant du verre dopé au néodyme produisaient des impulsions de quelques centaines de femtosecondes, à des cadences de 10 à $50 \mathrm{kHz}$ et des énergies de $25 \mu \mathrm{J}$. Ces lasers, basés sur la technique CPA et sur des amplificateurs régénératifs, restaient encombrants et complexes selon les critères actuels, mais suffisamment petits néanmoins pour permettre leur intégration dans un bloc opératoire. L'idée première était d'effectuer la découpe de la petite lentille dans la cornée grâce à la possibilité de focaliser le laser femtoseconde au sein d'un milieu transparent, puis de l'extraire au travers d'une petite ouverture qui serait effectuée sur le côté de la cornée. Malheureusement, la précision de découpe nécessaire à une bonne correction restait à l'époque difficile à atteindre et ce n'est que très récemment que la société Zeiss a mis au point la technique dite SMILE qui utilise cette idée et permet donc une correction de la myopie entièrement par laser femtoseconde.

En pratique, il est encore trop complexe de traiter tous les cas par cette technique et la plupart des opérations de la myopie sont effectuées en utilisant le laser femto- seconde seulement pour découper le capot. Une couche plane de bulles d'environ $5 \mu \mathrm{m}$ d'épaisseur et à environ $100 \mu \mathrm{m}$ sous la surface de la cornée est créée, permettant de former le capot, qui est ensuite soulevé par le chirurgien. Un laser nanoseconde à excimère est alors utilisé pour sculpter la cornée, et le capot est finalement repositionné afin de protéger la surface de l'œil. Le laser femtoseconde n'intervient donc que très ponctuellement dans cette opération, mais cela n'a pas empêché des milliers de systèmes d'être vendus et près de 2 millions d'opérations d'être effectuées chaque année avec cette technique. Elle est devenue, essentiellement pour des raisons de marketing, la méthode de référence de la correction de la myopie par laser.

\section{Opération de la cataracte}

Le défi suivant auquel vont s'attaquer les lasers femtoseconde est l'opération de la cataracte, qui consiste à retirer le cristallin de l'œil et à le remplacer par un implant en plastique. C'est l'acte chirurgical le plus pratiqué, avec 25 millions d'opérations par an dans le monde. Cette opération est effectuée manuellement par un chirurgien, à l'aide d'un couteau et d'une pince. Le laser femtoseconde permet de remplacer le couteau pour découper précisément la capsule qui entoure le cristallin (opération appelée "capsulorhexis") et de prédécouper le cristallin avant son extraction. La communauté des chirurgiens reconnait l'apport notable du laser dans la précision du capsulorhexis et la diminution du risque de découpe ratée. Par ailleurs, les opérations actuelles se cantonnent, dans l'immense majorité des cas, à un implant monofocal donnant une vision corrigée à une distance fixe, généralement l'infini. Avec le laser femtoseconde, la découpe est parfaitement maitrisée et on peut alors envisager l'utilisation des nouveaux implants multifocaux, fonctionnant comme les verres progressifs de lunettes, qui nécessitent un positionnement très précis. Néanmoins le surcout lié au laser femtoseconde reste trop important pour cette opération et, actuellement, seuls quelques systèmes sont installés, essentiellement aux USA.

Les lasers femtoseconde ont donc révolutionné certains actes de chirurgie en ophtalmologie, mais l'immense marché de la cataracte reste d'un accès compliqué à cause du cout des lasers. Il reste encore à inventer, trente ans après l'introduction du CPA, la façon de produire des impulsions femtosecondes à des couts raisonnables. 


\section{Intensités fortes:}

\section{applications à la recherche}

\section{Les aspects technologiques}

Catherine Le Blanc (catherine.leblanc@polytechnique.fr)

Laboratoire pour I'Utilisation des Lasers Intenses (CNRS/École polytechnique/CEA/Sorbonne Université),

École polytechnique, 91128 Palaiseau Cedex

Les lasers de très haute intensité se composent de quatre grandes parties (fig. III.1) : une première partie "pilote " avec un oscillateur ultracourt et un étireur d'impulsion (voir l'article de F. Druon, p. 14), une deuxième partie avec des amplificateurs de puissance de tailles croissantes pompés par des lasers de très fortes énergies ; la troisième partie est le compresseur d'impulsion comprenant des grands réseaux de diffraction installés sous vide, et la dernière est le transport sous vide du faisceau et sa focalisation. Pour atteindre des intensités de plus de $10^{22} \mathrm{~W} \mathrm{~cm}^{-2}$, il faut non seulement avoir des impulsions de fortes énergies (plusieurs centaines de joules), de durées très courtes (inférieures à $20 \mathrm{fs}$ ), mais aussi que le faisceau soit focalisable sur une petite surface (quelques microns carrés). Tous ces paramètres deviennent difficiles à atteindre quand on va vers ces extrêmes, et on est très vite rattrapé par les limites de la technologie. Dans ce contexte, le projet français Apollon $10 \mathrm{PW}$ (soit $180 \mathrm{~J}$, $18 \mathrm{fs}$ ) est un très bon exemple d'installation laser de très haute intensité qui est à la frontière de la technologie [10, 14]. Pour illustrer les défis rencontrés, nous évoquerons tout d'abord l'amplification avec le saphir dopé au titane $\left(\mathrm{Ti}^{3+}: \mathrm{Al}_{2} \mathrm{O}_{3}\right.$ noté ici $\mathrm{Ti}$ : Sa), milieu amplificateur qui a permis d'atteindre des records de puissance crête, puis la technologie liée à l'étirement et à la compression d'impulsions à très large spectre et à très haute énergie, et enfin la gestion de la focalisation du faisceau sur des tailles les plus petites possible.

Le premier défi concerne donc l'amplification et plus particulièrement les milieux amplificateurs. On peut identifier deux grandes classes de milieux amplificateurs utilisés pour les lasers intenses : le verre dopé au néodyme ( $\mathrm{Nd}$ : verre) et le cristal Ti : Sa. Les lasers Nd : verre ont été les premiers à être utilisés en mode CPA [1] en 1985, jusqu'à atteindre le pétawatt au
Laurence Livermore National Laboratory [15] en 1999. Dans les années 1990 est apparu le Ti : Sa, qui s'est révélé être le matériau le plus propice aux impulsions ultracourtes et intenses. Avec ses $200 \mathrm{~nm}$ de largeur spectrale, sa conductivité thermique élevée, sa capacité à stocker beaucoup d'énergie sur une petite surface (fluence de saturation élevée), il est le meilleur candidat pour atteindre des puissances crêtes élevées.

Ainsi, en utilisant des cristaux de taille suffisamment grande $(200 \mathrm{~mm}$ de diamètre pour le $\mathrm{Ti}$ : Sa) (fig. III.2b), on sera capable d'extraire des énergies de plusieurs centaines de joules. Mais la difficulté ici est la fabrication de ce cristal en très grande taille. Depuis plus de vingt ans, de nombreux industriels ont travaillé sur la production de grands cristaux, homogènes en surface et en volume, mais souvent sans succès. Aujourd'hui, seule la société américaine GTAT peut fabriquer des cristaux

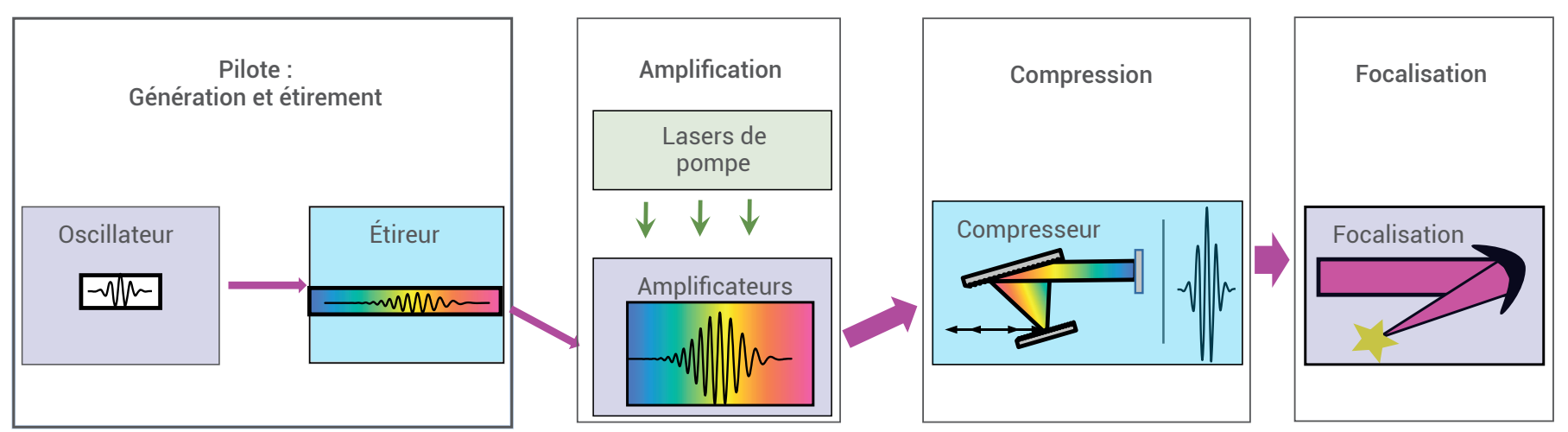

III.1. Principe d'une chaine d'amplification CPA, avec quatre grands blocs : le pilote, les amplificateurs de puissance, le compresseur d'impulsion et le système de focalisation. 
de Ti : Sa de plus de $200 \mathrm{~mm}$ de diamètre - ce qui permet toutefois d'extraire plus de 300 joules. Le laboratoire SIOM en Chine est aussi depuis peu un fournisseur potentiel de cristaux de grande taille ; reste à en vérifier la qualité et la reproductibilité.

Le deuxième défi qui, dans ce contexte CPA, prend toute sa signification est la maitrise de l'étirement et de la compression des impulsions très courtes et énergétiques. La difficulté est d'étirer les impulsions très courtes sur cinq ordres de grandeur (de 10 fs à 1 ns), tout en gardant l'intégralité de la bande spectrale et sans y ajouter d'aberration optique (ni chromatisme ni aberration sphérique ou coma), sous peine de ne pas pouvoir recomprimer les impulsions à leur durée initiale. La technique expliquée précédemment (voir l'article de F. Druon, p.14) est ici optimisée à l'extrême, et l'étireur de type Offner [16] qui n'utilise que des optiques réflectives (fig. III.2a) est une très bonne solution pour étirer sans aberration des impulsions courtes. La compression de l'impulsion est aussi une partie délicate de la technique CPA. L'énergie en sortie des amplificateurs est très élevée (300 J sur l'installation Apollon) et les réseaux utilisés pour la compression ont la fâcheuse caractéristique d'avoir un seuil d'endommagement très bas (autour de $400 \mathrm{~mJ} \mathrm{~cm}^{-2}$ ). Depuis la démonstration du CPA, la technique de fabrication des réseaux n'a pas fondamentalement évolué. Même si depuis plusieurs années, on a vu apparaitre des réseaux avec des traitements multicouches diélectriques plus efficaces et plus robustes, leur acceptance spectrale, c'est-à-dire la gamme de longueurs d'onde
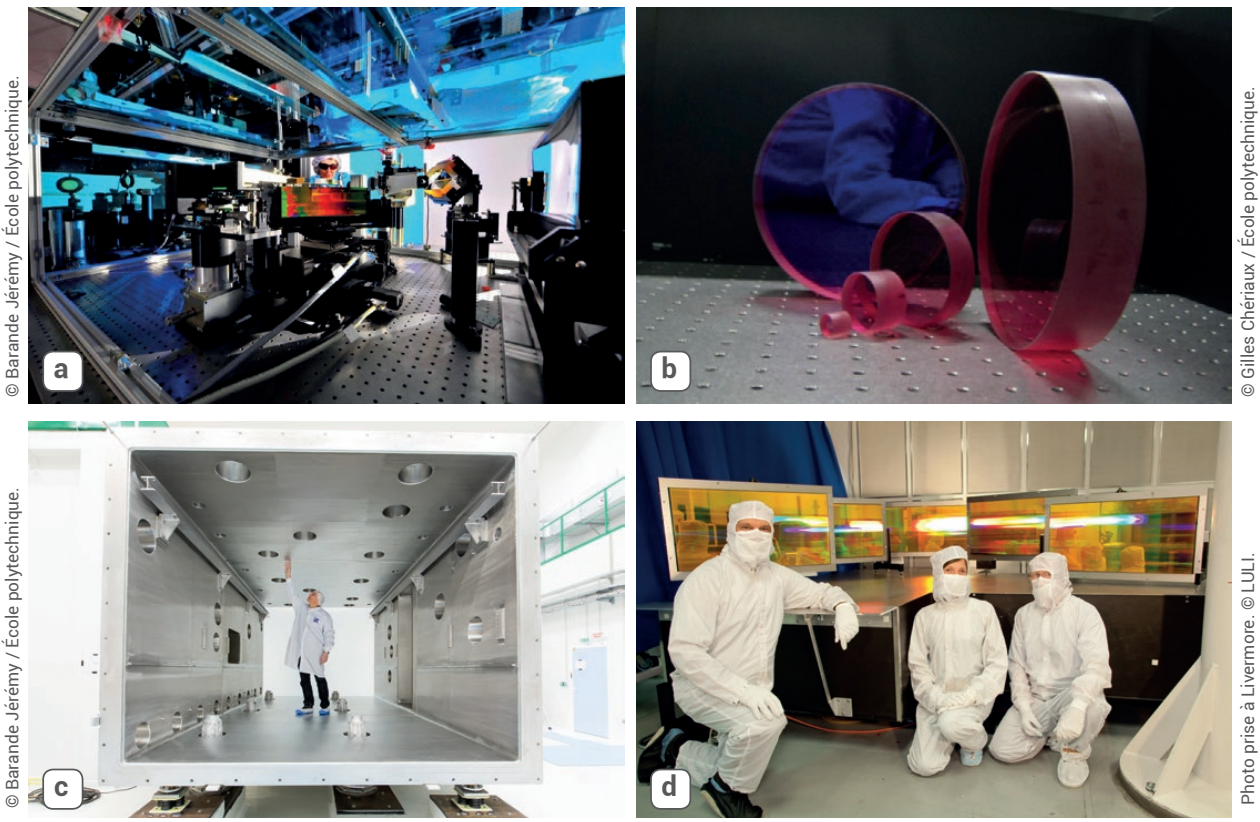

III.2. Photos de l'installation 10 PW Apollon. (a) Étireur Offner à deux réseaux. (b) Cristaux amplificateurs de Ti : Sa (le plus grand mesure $200 \mathrm{~mm}$ de diamètre). (c) Enceinte à vide du compresseur de 6 mètres de long et $3 \mathrm{~m}$ de haut, en cours de montage. (d) Réseaux métriques en or (940 x $480 \mathrm{~mm})$ pour la compression des impulsions.

dans laquelle le réseau peut travailler, n'est pas suffisamment large pour des impulsions ultracourtes. Aujourd'hui, les seuls réseaux commerciaux capables de recomprimer des impulsions sub-50 fs sont les réseaux recouverts d'une couche d'or. Les fabricants - comme par exemple Horiba France SA - ont toutefois amélioré considérablement leur technique et fournissent aujourd'hui des réseaux extrêmement efficaces (94\% d'efficacité de diffraction) sur des tailles pouvant atteindre un mètre, avec une excellente qualité de front d'onde. Pour pallier la faible tenue au flux des réseaux, les faisceaux énergétiques à la sortie des amplificateurs doivent être agrandis d'un facteur 3 environ. Dans le cas du laser Apollon, le faisceau est élargi à $400 \mathrm{~mm}$ de diamètre et est comprimé par quatre réseaux en or d'un mètre de longueur (fig. III.2d). La compression se réalise dans une grande chambre à vide (fig. III.2c), afin d'éviter les effets non linéaires lors de la propagation de l'impulsion intense.

Le troisième défi concerne la capacité à focaliser le faisceau. En sortie du compres- seur, ce dernier est dirigé vers une chambre d'interaction et est focalisé pour interagir avec le milieu solide ou gazeux. Pour cela, on utilise couramment des miroirs paraboliques hors axe de très grande ouverture. De plus, pour permettre une focalisation optimum, des miroirs déformables sont placés à l'air avant le compresseur. Ces miroirs, composés d'actuateurs actifs répartis sur toute la surface, sont capables de corriger les déformations de front d'onde subies par le faisceau au cours de l'amplification [17]. En général, la mesure des aberrations est réalisée en sortie du dernier amplificateur, et la correction adéquate est appliquée au miroir déformable.

Tous ces facteurs réunis permettent aujourd'hui de développer des installations laser capables de fournir des puissances crêtes de plus de $10 \mathrm{PW}$, avec des intensités de $10^{23} \mathrm{~W} \mathrm{~cm}{ }^{-2}$ (voir l'article de P. Zeitoun, p. 23). Le laser Apollon [18] ouvrira ses portes aux utilisateurs en 2019, en délivrant $1 \mathrm{PW}$ dans une première phase, et pourra à partir de 2020 atteindre son niveau attendu de $10 \mathrm{PW}$. 


\title{
Les impulsions ultracourtes
}

\author{
Pascal Salières (pascal.salieres@cea.fr) et Fabien Quéré \\ Laboratoire Interactions, Dynamiques et Lasers (CEA/CNRS/Université Paris-Saclay) \\ CEA Saclay, 91191 Gif-sur-Yvette Cedex
}

La compréhension des processus intervenant au cœur de la matière, à l'échelle atomique, est devenue fondamentale dans notre société basée sur la haute technologie, car elle ouvre la porte à leur contrôle. Lorsqu'on considère des systèmes de plus en plus petits, les temps caractéristiques des mouvements deviennent de plus en plus courts et requièrent des techniques d'observation de plus en plus rapides, tels que des flashs de lumière ultrabrefs capables de "photographier " l'état transitoire du système sans flou artistique. C'est ainsi que l'invention des lasers femtoseconde dans les années 1980 a permis l'observation des vibrations moléculaires : la femtoseconde est précisément l'échelle caractéristique du mouvement des atomes dans les molécules. L'importance des développements de la "femtochimie " a valu le Prix Nobel à Ahmed Zewail en 1999 [19]. Franchir une nouvelle étape dans l'exploration de la matière, c'est aller observer les mouvements des électrons dans les atomes, les molécules ou les solides, qui se déroulent à une échelle de temps encore plus extrême : l'attoseconde (as) [20]. Comment produire des flashs de lumière d'une telle brièveté ? Le lien de transformée de Fourier existant entre temps et fréquences nous enseigne qu'il faut pour cela un très large spectre s'étendant dans l'extrême ultraviolet (UVX) et dont les différentes fréquences soient toutes en phase, donc un rayonnement UVX cohérent de très large bande [21]. Une solution pour obtenir un tel rayonnement a été identifiée dans les années 1990 : il s'agit d'induire un élargissement considérable du spectre d'une impulsion laser en utilisant des effets optiques fortement non linéaires, plus précisément en générant tout un ensemble d'harmoniques d'ordre très élevé de la fréquence laser. Comme ces harmoniques conservent en grande partie la cohérence du laser fondamental, les conditions de Fourier sont alors réunies. Les premières expériences de ce type ont
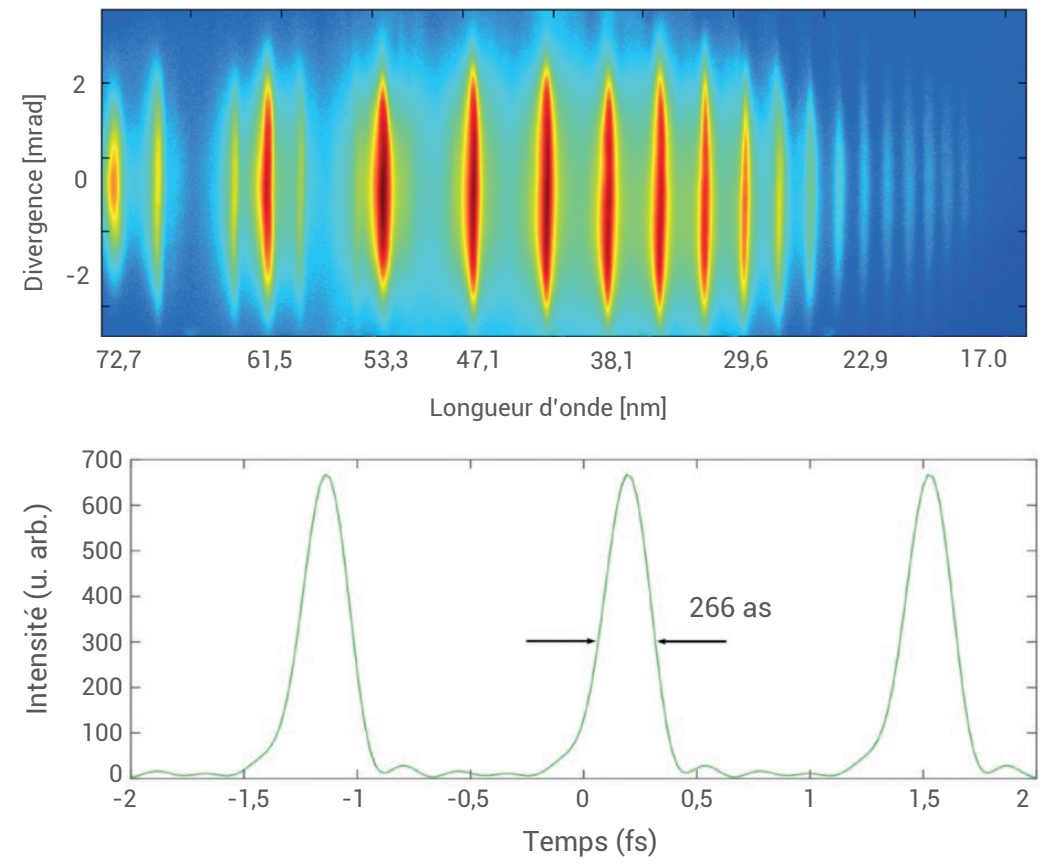

III.3. Émission de flashs attosecondes de rayonnement UVX. (a) Spectre des harmoniques 11 à 47 générées en focalisant un laser Ti : Sa à un éclairement de $210^{14} \mathrm{~W} \mathrm{~cm}^{-2}$ dans un gaz d'argon sur I'installation ATTOLab [28]. (b) Train d'impulsions de 266 as correspondant à la gamme spectrale couvrant les harmoniques 13 à 23.

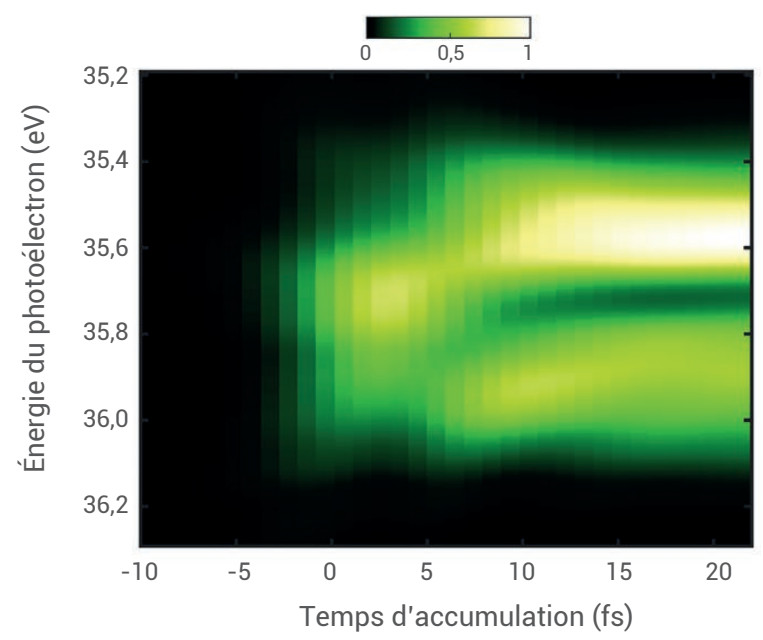

III.4. Évolution temporelle du spectre d'énergie des électrons émis après excitation d'un atome d'hélium avec des impulsions de 266 as centrées à $60 \mathrm{eV}$. Un profil dissymétrique apparait environ 5 fs après l'absorption des photons UVX. Ce profil résulte de l'interférence entre deux voies possibles d'éjection de l'électron, l'une directe, l'autre passant par un piégeage transitoire dans un état excité (résonance 2s2p de durée de vie 17 fs). On observe ici directement en temps un phénomène prédit théoriquement en 1961 par le physicien italien Ugo Fano. 
utilisé des lasers à impulsions "longues " (30 ps) [22], mais c'est la technique CPA qui a permis d'obtenir des impulsions laser suffisamment brèves (fs) et intenses pour à la fois générer des ordres harmoniques très élevés (plusieurs centaines), et confiner l'émission à une impulsion attoseconde isolée. C'est ainsi que les impulsions les plus brèves à l'heure actuelle ont pu être générées (43 as à l'ETH Zurich en 2017). Deux processus physiques distincts de génération d'harmoniques élevées ont été démontrés à ce jour, dans des gammes d'intensité très différentes. La génération d'harmoniques dans les gaz atomiques et moléculaires intervient lorsqu'on focalise des impulsions laser à des éclairements dans la gamme $10^{14}-10^{15} \mathrm{~W} \mathrm{~cm}^{-2}$ [23]. Lorsque le champ laser oscillant atteint un maximum, il peut arracher par effet tunnel des électrons et les réaccélérer vers leurs ions parents une demi-période plus tard quand il change de signe ; cette " recollision " peut donner lieu à une recombinaison radiative de l'électron avec son ion parent, résultant en l'émission de flashs attosecondes de rayonnement UVX [21] (fig. III.3). La génération d'harmoniques dans les plasmas met quant à elle en jeu des intensités laser allant de $10^{16} \mathrm{~W} \mathrm{~cm} \mathrm{~cm}^{-2}$ jusqu'aux intensités les plus élevées (estimées à $10^{22} \mathrm{~W} \mathrm{~cm}^{-2}$ ) disponibles à ce jour grâce à la technique CPA. Différents mécanismes peuvent donner lieu à cette génération [24]. Aux intensités les plus élevées, la surface réfléchissante du plasma créé par le champ laser à la surface d'une cible solide - appelée miroir plasma oscille à vitesse relativiste sous l'effet du champ laser ultra-intense. Ce miroir oscillant relativiste induit un effet Doppler périodique sur le faisceau laser réfléchi, qui comprime temporellement certaines parties du champ laser, produisant ainsi des impulsions attosecondes.

Depuis les premières mesures en 2001 [24], de nombreuses applications ont été effectuées. Sonder la matière avec des impulsions attosecondes donne par exemple accès aux temps de diffusion des électrons dans les atomes, les molécules ou les solides, comme illustré récemment par l'observation en temps réel de l'émission photo-induite d'électrons par des atomes [26] (fig. III.4) ou des molécules [27]. Les impulsions attosecondes ouvrent la perspective de contrôler les processus à une échelle longtemps inaccessible, depuis la réactivité chimique pour la fabrication de nouvelles molécules jusqu'aux courants dans les nanocircuits pour l'optoélectronique ultrarapide.

\title{
L'ultra-haute intensité
}

\author{
Philippe Zeitoun (philippe.zeitoun@ensta-paristech.fr) \\ Laboratoire d'Optique Appliquée (CNRS/ENSTA-ParisTech/École polytechnique), 91762 Palaiseau Cedex
}

Depuis la fin des années 1990, les lasers intenses et ultra-brefs ont permis de réaliser des progrès fulgurants sur l'accélération de particules par laser et de générer des sources secondaires d'électrons, de protons et également de rayonnement X et $\gamma$. Bien qu'il soit délicat de donner une limite au régime d'ultra-haute intensité, il est communément admis que celle-ci correspond au régime relativiste, c'est-à-dire à des intensités suffisantes pour accélérer des électrons à des énergies relativistes, donc avec une énergie cinétique, $\mathrm{E}_{\mathrm{c}}$, au moins équivalente à leur énergie au repos $\left(\mathrm{E}_{\mathrm{m}}=512 \mathrm{keV}\right)$. Cette limite se situe vers $10^{18} \mathrm{~W} \mathrm{~cm}^{-2}$ (fig. III.5) et n'a pu être atteinte dans les années 1990 que grâce à la technique du CPA. La course aux intensités extrêmes a vu la construction des très grands lasers ultra-brefs comme HERCULES aux États-Unis ${ }^{(a)}$, KAIST en Corée, auxquels vont s'ajouter les projets de laser $10 \mathrm{PW}$ lancés par Gérard
Mourou : APOLLON en France et les trois piliers de ELI (Extreme Light Infrastructure), en Hongrie (dédié à la génération d'impulsions attosecondes), en République tchèque (dédié à la génération de sources secondaires) et en Roumanie (dédié à la physique nucléaire), jusqu'au projet SEL de laser 100 PW en Chine (fig. III.5).

Historiquement, l'accélération d'électrons par laser a été proposée par Toshiski Tajima et John M. Dawson en 1979 [29] et a débuté avec de faibles accélérations en produisant des électrons de $9 \mathrm{MeV}$ [30]. Un plasma est produit par l'interaction d'un laser ultra-intense avec un gaz et, sous l'effet des champs de l'onde laser, une séparation longitudinale des charges se crée (onde de sillage [10]), produisant un champ électrique très intense pouvant atteindre plusieurs dizaines de $\mathrm{GV} \mathrm{m}^{-1}$ et se déplaçant à une vitesse proche de celle de la lumière, dans lequel les électrons peuvent être accélérés très fortement.
Aujourd'hui, le record atteint de l'accélération par laser est de $4 \mathrm{GeV}$ avec quelques centimètres de gaz, contre une centaine de mètres pour les meilleurs accélérateurs conventionnels. Différents nouveaux concepts d'accélération laser laissent entrevoir la possibilité d'atteindre $100 \mathrm{GeV}$, voire le TeV pour des longueurs d'accélération de quelques mètres au lieu de plusieurs dizaines de kilomètres aujourd'hui. Cependant, les faisceaux d'électrons accélérés par laser ont pour l'instant une charge faible (nombre d'électrons dans un paquet), une grande dispersion en énergie et ne sont pas assez stables. Des travaux d'ingénierie doivent être menés pour faire passer cette technique de la démonstration scientifique à un outil pour les physiciens et pour les applications.

On atteint le régime ultra-relativiste quand les intensités sont suffisantes pour accélérer les protons $\left(\mathrm{E}_{\mathrm{m}} \approx 938 \mathrm{MeV}\right)$ à des énergies relativistes (fig. III.5). La limite se 


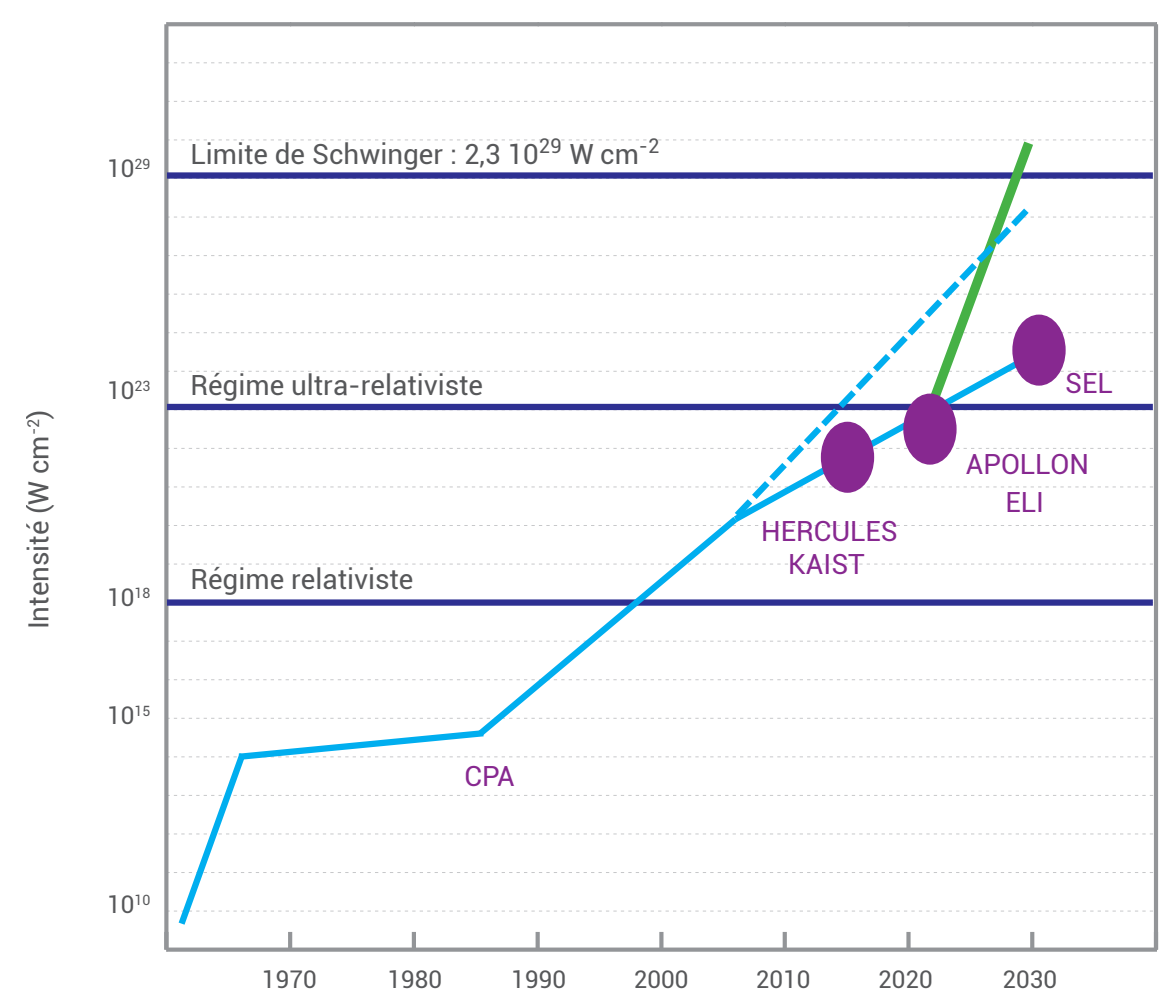

III.5. La route vers les très hautes intensités depuis les premiers lasers des années 1960. Intensités laser avant et après le CPA (en bleu) et projection pour des faisceaux X focalisés ultra-intenses (en vert). HERCULES (États-Unis, 0,3 PW) et KAIST (Corée, 3 PW) sont des installations opérationnelles, APOLLON (France) et ELI (Europe) visent les 10 PW, et SEL (Chine) est un projet à 100 PW.

situe vers $10^{23} \mathrm{~W} \mathrm{~cm}^{-2}$. Même si ces intensités n'ont pas encore été atteintes, l'accélération de protons (et autres hadrons) est une thématique de recherche très active sur des lasers délivrant des intensités supérieures à $10^{20} \mathrm{~W} \mathrm{~cm} \mathrm{~cm}^{-2}$. L'interaction du laser avec un solide ou avec un gaz à très haute pression produit, par séparation des charges, des champs électriques très élevés, de l'ordre du TV $\mathrm{m}^{-1}$, qui sont nécessaires pour accélérer les protons [10]. De plus, vers une intensité de $10^{23} \mathrm{~W} \mathrm{~cm}^{-2}$, les simulations numériques prédisent l'apparition d'un nouveau régime où des feuilles ultraminces $(10 \mathrm{~nm})$ peuvent être accélérées directement par la pression de la lumière laser. Ces jets de protons relativistes et de très brèves durées (sub-ps) semblent intéressants, en particulier en physique nucléaire pour exciter des noyaux et les placer dans des états inconnus ou peu explorés, ou encore pour produire des sources de neutrons de très grande brillance instantanée par spallation $^{(b)}[31]$.

Par ailleurs, sous certaines conditions, les électrons accélérés par laser dans un gaz produisent une séparation transverse des charges positives et négatives ce qui, par contre-réaction, les pousse à osciller. Le rayonnement produit par cette oscillation relativiste est un cône de rayons $\mathrm{X}$ : c'est ce que l'on appelle l'effet bétatron [32]. Ce rayonnement $\mathrm{X}$ a un spectre large comme celui qui est produit dans les synchrotrons, avec une durée d'émission de l'ordre de la femtoseconde. Comme précédemment, c'est la très faible longueur d'accélération (quelques millimètres ici) qui rend ce système très compact par rapport aux synchrotrons ou aux lasers à électrons libres X (X-FEL). Les applications sont encore à leurs prémices, mais la durée extrêmement courte de cette émission ouvre des perspectives importantes en physique fondamentale, en science des matériaux et en radiobiologie (voire un jour en médecine), par exemple en réalisant des films à cadence ultrarapide avec des résolutions spatiales atomiques.

Il est naturel de rêver d'atteindre la prochaine frontière à encore plus haute intensité et le domaine de l'électrodynamique quantique (QED) non linéaire (fig. III.5). Pour des intensités de $10^{22} \mathrm{~W} \mathrm{~cm}^{-2}$ et au-delà, le laser doit induire une polarisation du vide détectable. Des concepts très fondamentaux de QED non linéaire, comme la réaction quantique du rayonnement émis par une particule chargée, peuvent être démontrés au-delà de $10^{23} \mathrm{~W} \mathrm{~cm}^{-2}$. Le test ultime en QED non linéaire consiste à perturber le vide à l'aide d'un laser ultra-intense, afin de créer des paires électron-positron. Il faudrait alors atteindre une intensité de $2,310^{29} \mathrm{~W} \mathrm{~cm}^{-2}$, appelée intensité de Schwinger [33]. Pour un laser visible ou infrarouge ultime, c'està-dire ayant une largeur temporelle ultime d'une "période " (durée de 2,7 fs pour un laser Ti : Sa) et focalisé en limite de diffraction (tache focale de $2 \mu \mathrm{m}$ ), son énergie devrait être d'environ $20 \mathrm{MJ}$, soit 100000 lasers de type ELI ou APOLLON. Une solution à cette apparente impossibilité a été apportée par Gérard Mourou et ses collaborateurs, en proposant d'utiliser un faisceau de rayons $\mathrm{X}$ cohérent produit par la technique de miroirs relativistes décrite précédemment (voir l'article de P. Salières et F. Quéré, p.22). Ici, c'est l'association de cette technique avec des impulsions de largeur temporelle ultime qui donne l'efficacité nécessaire pour obtenir un faisceau de rayons $X$ très énergétique [34]. Le changement de longueur d'onde a deux effets majeurs : réduction de la durée $(<50$ as) et possibilité d'obtenir des taches focales 100 fois plus petites que dans le visible. Une telle impulsion de rayons X de forte énergie, très courte et très bien focalisée, devrait permettre de réaliser un saut de géant en intensité et ainsi de dépasser l'intensité de Schwinger de 2,310 ${ }^{29} \mathrm{~W} \mathrm{~cm}^{-2}$ (trait vert de la figure III.5). Avec les avancées récentes sur la génération d'harmoniques sur solide, sur les optiques $\mathrm{X}$, en particulier pour la lithographie à $13,5 \mathrm{~nm}$, et sur les mesures de front d'onde X, cette expérience de pensée semble réalisable d'ici moins de 10 ans.

Avec le CPA et avec sa volonté d'aller toujours plus loin, Gérard Mourou n'a cessé d'ouvrir la voie de l'exploration des intensités laser de plus en plus élevées et, avec elles, de nombreux champs d'expériences et de recherche pour les générations présentes et futures.

(a) Au CUOS, Center for Ultrafast Optical Science, fondé par Gérard Mourou en 1990.

(b) La spallation est une réaction nucléaire au cours de laquelle un noyau atomique est frappé par une particule incidente et se fragmente en noyaux plus légers et des particules. 
1 D. Strickland et G. Mourou, "Compression of amplified chirped optical pulses", Opt. Commun. 56 (1985) 219.

2. P. Tournois, "Analogie optique de la compression d'impulsion ", Compt. Rend. Acad. Sci. (Paris) 258 (1964) 3839 ; P. Tournois, "Analogie optique de la compression d'impulsion ", Ann. Radioelec. 19 (1964) 267 ; P. Tournois et al., « Sur l'analogie optique de certains montages électroniques : Formation d'images temporelles de signaux électriques ", Compt. Rend. Acad. Sci. (Paris) 267 (1968) 375.

3. E. B. Treacy, "Optical pulse compression with diffraction gratings", IEEE J. Quantum Electron. 5 (1969) 454.

4 C. Sauteret et al., "Generation of 20-TW pulses of picosecond duration using chirped-pulse amplification in a Nd:glass power chain", Opt. Lett. 16 (1991) 238.

5• M. D. Perry et al., "Petawatt laser pulses", Opt. Lett. 24 (1999) 160.

6• 0. E. Martinez et al., "Negative group-velocity dispersion using refraction", J. Opt. Soc. Am. A 1 (1984) 1003.

7•P. F. Moulton, "Spectroscopic and laser characteristics of Ti: $\mathrm{Al}_{2} \mathrm{O}_{3}$ ", J. Opt. Soc. Am. B 3 (1986) 125.

8. C. Horvath et al., "Compact directly diode-pumped femtosecond Nd:glass chirped-pulse-amplification laser system", Opt. Lett. 22 (1997) 1790

9- T. Juhasz et al., "Femtosecond Laser Refractive Corneal Surgery", IEEE Journal of Special Topics in Quantum Electronics 5 (1999) 902.

10 F. Amiranoff, «Apollon: le laser de l'extrême », Reflets de la Physique 47-48 (2016) 60

11 - A. P. Joglekar et al., "A study of the deterministic character of optical damage by femtosecond laser pulses and applications to nanomachining", Appl. Phys. B 77 (2003) 25.

12• A. P. Joglekar et al., "Optics at critical intensity: Applications to nanomorphing", PNAS 101 (2004) 5856.

13• F. H. Loesel et al., "Ultraprecise medical applications with ultrafast lasers: corneal surgery with femtosecond lasers", Proc. SPIE 3564, Medical Applications of Lasers in Dermatology, Cardiology, Ophthalmology, and Dentistry II (1999).

14 J. P. Zou et al., "Design and current progress of the Apollon $10 \mathrm{PW}$ project", High Power Laser Science and Engineering 3 (2015) e2.

15• M. D. Perry et al., "Petawatt Laser Pulses", Opt. Lett. 24 (1999) 160.

16 G. Chériaux et al., "Aberration-free stretcher design

for ultrashort-pulse amplification", Opt. Lett. 21 (1996) 414.
17• K. Nakamura et al., "Diagnostics, control and performance parameters for the BELLA high repetition rate petawatt class laser", IEEE Journal of Quantum Electronics 53 (2017) 1200121.

18・ www.apollon-laser.fr

19• A. Zewail, "Femtochemistry: Atomic-Scale Dynamics of the Chemical Bond", J. Phys. Chem. A 104 (2000) 5660.

20- P. B. Corkum et F. Krausz, "Attosecond science", Nature Physics 3 (2007) 381.

21 • T. Ruchon et al., "Sources cohérentes de laboratoire dans l'extrême ultraviolet », Reflets de la Physique 21 (2010) 30.

22 M. Ferray et al., "Multiple-harmonic conversion of $1064 \mathrm{~nm}$ radiation in rare gases", J. Phys. B 21 (1988) L31.

23• F. Krausz et M. Ivanov, "Attosecond physics", Rev. Mod. Phys. 81 (2009) 163

24 - F. Quéré et P. Martin, « Vers l'optique à ultra-haute intensité : l'exemple des miroirs plasmas ", Reflets de la Physique 19 (2010) 14

25 P. M. Paul et al., "Observation of a train of attosecond pulses from high harmonic generation", Science 292 (2001) 1689. M. Hentschel et al., "Attosecond metrology", Nature 414 (2001) 509.

26• V. Gruson et al., "Attosecond dynamics through a Fano resonance: Monitoring the birth of a photoelectron", Science 354 (2016) 734.

27- S. Beaulieu et al., "Attosecond-resolved photoionization of chiral molecules", Science 358 (2017) 1288

28・ http://attolab.fr/

29• T. Tajima et J. M. Dawson, "Laser electron accelerator", Phys. Rev. Lett. 43 (1979) 267.

30 C.E. Clayton et al., "Ultra high-gradient acceleration of injected electrons by laser-excited relativistic electron plasma waves", Phys. Rev. Lett. 70 (1993) 37.

31 - J. Fuch et al., "Laser-driven proton scaling laws and new paths towards energy increase", Nat. Phys. 2 (2006) 48.

32- A. Rousse et al., "Production of a keV x-ray beam from synchrotron radiation in relativistic laser-plasma interaction", Phys. Rev. Lett. 93 (2004) 135005.

33. J. Schwinger, "On gauge invariance and vacuum polarization", Phys. Rev. 82 (1951) 664.

34- N. M. Naumova et al., "Isolated attosecond pulses generated by relativistic effects in a wavelength-cubed focal volume", Opt. Lett. 29 (2004) 778. 